\title{
Managing a gastrointestinal oncology practice in Japan during the COVID-19 pandemic: single institutional experience in The Cancer Institute Hospital of Japanese Foundation for Cancer Research
}

\author{
Daisuke Takahari ${ }^{1}$ (D) Eiji Shinozaki ${ }^{1} \cdot$ Takeru Wakatsuki $^{1} \cdot$ Akira Ooki $^{1} \cdot$ Masato Ozaka $^{1} \cdot$ Takeshi Suzuki $^{1,2}$. \\ Izuma Nakayama $^{1}$ - Hiroki Osumi ${ }^{1}$ - Daisaku Kamiimabeppu ${ }^{1} \cdot$ Taro Sato $^{1} \cdot$ Mariko Ogura $^{1} \cdot$ Mitsukuni Suenaga $^{1}$. \\ Keisho Chin $^{1} \cdot$ Kensei Yamaguchi ${ }^{1}$
}

Received: 15 June 2020 / Accepted: 4 October 2020 / Published online: 21 October 2020

(c) The Author(s) 2020

\begin{abstract}
Coronavirus disease 2019 (COVID-19) was declared to be a global pandemic by the World Health Organization on March 11, 2020. On April 7, 2020, a state of emergency was declared in Japan, as had been by other nations worldwide. This unprecedented crisis has profound implications for patients undergoing chemotherapy and for practicing healthcare professionals. Various reports have shown data indicating that cancer patients with COVID-19 have high morbidity and mortality rates. In order to reduce the use of medical resources to avoid the risk of COVID-19 infections in both cancer patients and health care providers, oncologists now have to draw the line for cancer treatments by maintaining their efficacy while avoiding severe adverse events. In this article, we outlined the decisions made regarding the practice of gastrointestinal oncology in our institution during the COVID pandemic.
\end{abstract}

Keywords Gastrointestinal oncology · COVID-19 · Chemotherapy

\section{Introduction}

A case of coronavirus disease 2019 (COVID-19), which is caused by the severe acute respiratory syndrome coronavirus 2 (SARS-CoV-2), was first reported in Wuhan City, China in December 2019. The World Health Organization (WHO) declared a global pandemic on March 11, 2020 [1]. As of May 20, 2020, there were 4,900,253 confirmed cases and 323,341 confirmed deaths worldwide [2]. The pandemic continues to spread over the globe. In Japan, as of 31 May, 2020, there were 16,851 cases of COVID-19 infection and 891 deaths [3]. On April 7, 2020, the Government of Japan declared a state of emergency under the Act on Special Measures for Pandemic Influenza and New Infectious

Daisuke Takahari

daisuke.takahari@jfcr.or.jp

1 Department of Gastroenterology, The Cancer Institute Hospital of Japanese Foundation for Cancer Research, 3-8-31 Ariake, Koto-ku, Tokyo 135-8550, Japan

2 Gastroenterology Department, Kawasaki Municipal Hospital, Kawasaki, Japan
Diseases Preparedness and Response. The areas where emergency measures were first implemented consisted of seven prefectures with the highest numbers of COVID-19 cases. However, on April 16, the emergency measures were expanded to include the entire country. The initial duration for the state of emergency was from April 7 to May 6, 2020, but then extended until May 31, 2020. Fortunately, it was lifted by May 25, but there is still a lot of caution to be taken. Even with the state of emergency, the government of Japan has worked to minimize its impact on social and economic functioning, and has not taken compulsory measures such as implementation of a "lockdown", which has been and is being implemented in other countries $[4,5]$. For cancer patients, of course, hospital visits for treatment and assessments are not subject to restriction, but the visits themselves can be a risk of infection, especially for individuals who have risk factors of COVID-19 infection. The basic principle of preventing infection with SARS-CoV-2 is to reduce close contact with other people.

Although evidence remains insufficient, several reports have indicated that cancer patients have significantly increased severity and complications associated with COVID-19 infection [6-8]. A meta-analysis found that the 
odds ratio of severe complications was 2.29 [95\% Confidence Interval (CI) 1.00-5.23] for patients with cancer [9]. The data suggest that patients with cancer more likely have severe disease leading to the need for intensive care and mechanical ventilation. And also, cancer patients with COVID-19 have high mortality rates [10-15]. The odds ratio of COVID-19 for death in patients with cancer and COVID19 was reported to be 2.93 (95\% CI 1.34-6.41, $p=0.006$ ) [12]. For this reason, cancer societies around the world have issued guidelines for cancer patients and cancer treatment during the COVID-19 pandemic. The American Society of Clinical Oncology (ASCO) has issued a statement on general care for clinicians, the cancer care delivery team, and patients with cancer during the COVID-19 pandemic [16]; and the European Society of Medical Oncology (ESMO) also has issued a statement of encouragement for medical oncologists as follows: (1) ensure the continuum of care, (2) be prepared for new routines, (3) protect yourself to protect your patients, and (4) reinforce support to patients [17]. In addition, several recommendations, including United States (US), French, and Italian guidelines were published [18-20]. In Japan, three societies [the Japanese Cancer Society, the Japanese Society of Clinical Oncology, and the Japanese Society of Medical Oncology (JSMO)] recently established a joint working group that issued the publication "New Coronavirus Infection and Cancer Care (Q\&A for Patients)" [21].

However, few specific recommendations on chemotherapy have been issued for patients with gastrointestinal (GI) cancers. Lou et al. recently proposed recommendations for minimizing risks to patients with GI cancers in the US [22].

The Cancer Institute Hospital of Japanese Foundation for Cancer Research is located in the center of Tokyo and is one of the largest cancer centers in Japan where provides approximately 20,000 GI cancer chemotherapy annually.

In this article, we present our clinical recommendations on how to modify the treatment but maintain effectiveness as much as possible for GI cancer patients receiving chemotherapies during the COVID-19 pandemic.

\section{Developing guidelines for patients with gastrointestinal cancers during the COVID-19 pandemic}

Several guidelines from the US and Europe on chemotherapy during the COVID-19 pandemic have been published $[16,17]$. The common strategies in these guidelines consist of the following: (1) balancing the benefits of treatment with risk of COVID-19 infection and exacerbation of cancer due to lack of treatment, and (2) reducing visits to the cancer center to reduce the risk of infection with SARS-CoV-2.

The risks of chemotherapy during the COVID-19 pandemic combined with the lack of guidance that takes into consideration the local circumstances in Japan have motivated us to develop a guidance for the use of chemotherapy in patients with GI cancers. The guidance was developed with the consensus of medical oncologists involved in GI cancer care in our institution (including 11 JSMOcertified oncology practitioners), who carefully considered whether the benefits of chemotherapy outweighed the risk of COVID-19 infection.

We examined the following questions:

1. What is the status of COVID-19 in Tokyo?

2. Does a particular therapy have enough evidence of clinical benefit to support it?

3. What are the considerations with regard to tumor burden and tumor growth rate?

4. Is the patient aged younger than 75 years with a performance status less than 2 ?

5. Are there any comorbidities such as hypertension, diabetic mellitus, cardiovascular/cerebrovascular disease, and chronic obstructive pulmonary disease that are relevant to the risk of COVID-19 severity?

6. How does the patient travel to our institution? Public or private transportation?

7. What is the patient's willingness to undergo treatment?

It was then discussed and finalized at each cancer multidisciplinary team conference with surgical and radiation oncologists, nurses, and pharmacists.

Table 1 shows the guidance on changes in treatments such as reducing the intensity of chemotherapy, delaying treatment, skipping cycles, or stopping according to the degree of severity of COVID-19 epidemic. Risk factors associated with COVID-19 are as follows: age ( $\geq 75$ years), performance status $\geq 2$, comorbidity (hypertension, diabetes mellitus, liver or renal or lung dysfunction). Low risk is defined as the presence of no risk factor. High risk is defined as the presence of one or more risk factors. Based on this concept, changes in treatments are considered for each type of GI cancer.

\section{General considerations}

Oncologists now have to draw the line for cancer treatments by maintaining their efficacy while avoiding severe adverse events and reducing the use of medical resources during the COVID-19 pandemic.

In accordance with ASCO recommendations [16], access to the institution is restricted to one entrance: all patients and visitors are screened there for fever, whether they have had contact with a patient with COVID-19, and screened for signs/symptoms (cough, dyspnea, and alterations in sense of smell or taste) of COVID-19. If the patient has had a fever greater than $37.5{ }^{\circ} \mathrm{C}$ during the previous 3 days or on the day of visit or any relevant signs/symptoms within 
Table 1 Changes in treatments according to the degree of severity of COVID-19 epidemic

\begin{tabular}{llll}
\hline Level & Degree of epidemic & Low risk $^{\mathrm{a}}$ & High risk $^{\mathrm{b}}$ \\
\hline 1 & Sporadic onset and mild increase of COVID-19 & No change & Consider \\
2 & Frequent onset and accelerating increase of COVID-19 & Consider & Recommend \\
3 & Spread of infection and overshoot of COVID-19 & Recommend & Recommend \\
\hline
\end{tabular}

Changes in treatments: Reducing the intensity of chemotherapy, delaying treatment, skipping cycles, or stopping

Risk factors associated with COVID-19 are: age ( $\geq 75$ years), performance status $\geq 2$, comorbidity (hypertension, diabetes mellitus, liver or renal or lung dysfunction). These recommendations are based on single institutional experience

${ }^{a}$ Low risk is defined as the presence of no risk factor

${ }^{\mathrm{b}} \mathrm{High}$ risk is defined as the presence of one or more risk factors
2 weeks, the patient will be transferred to a separate outpatient facility and undergo chest computed tomography (CT). If pneumonia is found or the attending physician deems it necessary after interviewing the patient, a polymerase chain reaction (PCR)-based SARS-CoV-2 assay of a patient specimen to detect the virus will be performed, and chemotherapy will not be administered on the same day, regardless of the patient's signs/symptoms. Unlike influenza, the severity of COVID-19 is associated with acute respiratory distress syndrome (ARDS) rather than secondary bacterial pneumonia [23]. Therefore, the neutropenia resulting from chemotherapy might not pose a risk of mortality in COVID-19; however, in general, immunosuppression should be avoided.

Except for the regimens of docetaxel, cisplatin, 5-fluorouracil (DCF) used in esophageal squamous cell carcinoma (ESCC), 5-fluorouracil, leucovorin, irinotecan, oxaliplatin (FOLFOXIRI) used in colorectal cancer (CRC), and FOLFIRINOX in pancreatic cancer (PC), severe immunosuppression is not commonly associated with the chemotherapy regimens used in GI oncology. Under low risk of COVID19 infection in the community, our routine clinical practice can usually follow established chemotherapy protocols. By contrast, for treating patients with high risk and/or during the pandemic, we must choose between reducing the intensity of chemotherapy (e.g., omission of the 5-FU bolus for CRC, replacement of infusional 5-FU by capecitabine or S-1 for ESCC, gastric cancer (GC), and CRC), delaying treatment, skipping cycles, or stopping (i.e., treatment maintenance phase and salvage chemotherapy with relatively few benefits). In addition, the empiric use of the prophylactic granulocyte colony-stimulating factor or antibiotics should be considered. We recommend the use of telemedicine for monitoring the side effects when treatment is delayed or chemotherapy cycles are skipped.

With regard to immunotherapy for GI cancers, immunecheckpoint inhibitor (ICI)s (nivolumab and pembrolizumab) can cause immune-related adverse events, including pneumonitis. We should accordingly weigh the potential clinical harm of immunotherapy in relation to its benefits, when considering its administration to a patient.

Furthermore, with regard to the use of anti-angiogenic agents (e.g., bevacizumab, ramucirumab, and aflibercept) for the treatment of GC and CRC, these agents are associated with a risk of arterial and venous thromboembolism, which have also been reported to occur at high rates in patients with COVID-19 [24, 25]. Although no evidence to avoid these anti-angiogenic agents during the pandemic is available, it is incumbent upon us to provide this information on COVID-19 to patients and healthcare providers. Moreover, hypertension is a frequent $\mathrm{AE}$ of antiangiogenic therapy, and antihypertensive drugs such as angiotensin II type 1 receptor blockers ARBs) or angiotensin-converting enzyme (ACE) inhibitors may affect expression of the ACE2, the receptor of SARS-Cov-2. So far, whether ACE inhibitors and ARBs worsen COVID-19-induced lung injury or not has been controversial $[26,27]$. Thus, there is no need to discontinue it at this time, but caution should be exercised in the future.

\section{Esophageal cancer}

\section{Neoadjuvant chemotherapy}

The standard neoadjuvant chemotherapy (NAC) used in Japan for clinical stage II/III ESCC is the combination of cisplatin plus 5-fluorouracil (CF) [28, 29]. A randomized controlled trial (JCOG9907) revealed its clinical benefit for curative resection and survival [28]. We should follow this principle of achieving curative resection and longterm survival, despite the COVID-19 pandemic. Several phase II studies [30, 31] found a clinical benefit for DCF in locally advanced ESCC. However, DCF, which is now being tested in the phase III trial JCOG1109, should not be recommended for NAC in clinical practice because of its hematological toxicity. In situations where surgery must be postponed or aborted because of various social situations, 
additional CF for NAC or changing treatment to definitive chemoradiation therapy should be considered.

\section{Adjuvant chemotherapy}

Adjuvant CF is indicated for patients in Japan with clinical stage II/III ESCC and with pathological nodal metastatic disease who received surgery without NAC [32]. However, the survival benefit of adjuvant $\mathrm{CF}$ was not definitive in the JCOG9204 trial [32]. Accordingly, adjuvant CF should be omitted during the COVID-19 pandemic.

\section{Palliative chemotherapy}

We also avoid DCF in the palliative setting because of the reasons listed in the previous section on 'Neoadjuvant chemotherapy'. With regard to palliative chemotherapy in the patients with high risk of COVID-19 infection, reduction in treatment intensity should be considered. Best supportive care can be an option, depending on the situation.

For first-line systemic chemotherapy, we might consider changing from $\mathrm{CF}$ to oral S-1 monotherapy. For second-line chemotherapy, nivolumab, which showed survival benefit in the ATTRACTION-3 trial [33], should be considered for administration with longer intervals between each administration. In addition, for patients receiving nivolumab for longer than 12 months, pausing its administration could be considered $[10,16]$. Taxanes such as paclitaxel or docetaxel can sometimes be candidates for third-line chemotherapy. Intervals between administration of these agents should also be longer, as the situation demands, because they sometimes cause severe neutropenia. Prophylactic granulocyte-colony stimulating factor should also be considered.

\section{Concurrent radiotherapy (RT)}

A total radiation dose of $60 \mathrm{~Gy}$ is often used for chemoradiotherapy in stage I or advanced ESCC [34, 35]. However, according to the RTOG regimen, 50.4 Gy of RT is generally used worldwide [36]. According to the RADS (Remote, Avoid, Defer, Shorten) principle [37], we have adopted $50 \mathrm{~Gy} / 25$ fractions (Fr) instead of $60 \mathrm{~Gy} / 30 \mathrm{Fr}$ to reduce the immunosuppressive effect of RT during the COVID-19 pandemic.

Table 2 lists the principles of chemotherapy for ESCC according to each risk group in the COVID-19 pandemic.

\section{Gastric cancer}

\section{Neoadjuvant chemotherapy}

NAC is not the standard treatment for resectable GC in Japan [38]. Thus, based on the results of the JCOG0405 trial [39], we only recommend NAC for resectable GC with bulky and/ or para-aortic lymph node metastases for patients with low risk of COVID-19 infection.

\section{Adjuvant chemotherapy}

Gastrectomy followed by adjuvant chemotherapy is the standard of care for patients with resectable GC in Japan [38]. The Adjuvant chemotherapy trial of S-1 for gastric cancer (ACTS-GC) trial verified the efficacy of S-1 monotherapy as adjuvant chemotherapy for Stage II or III GC [40, 41]. The Japan Clinical Cancer Research Organization (JACCRO)-GC 07 trial recently demonstrated that compared to S-1 monotherapy, the addition of docetaxel to S-1 (DS) significantly prolonged recurrence-free survival (RFS) for stage III GC [42]. Based on the results of these trials, S-1 monotherapy for Stage II and DS for Stage III GC patients is

Table 2 Principles of chemotherapy for esophageal cancer according to each risk group in the COVID-19 pandemic

\begin{tabular}{lll}
\hline Treatment settings & Low risk $^{\mathrm{a}}$ & High risk $^{\mathrm{b}}$ \\
\hline Neoadjuvant & CF & CF with dose reduction or 5-fluorouracil monotherapy \\
Adjuvant & Unrecommended & Unrecommended \\
Definitive chemoradiation & RT (50 Gy/25fr) plus CF & RT $(50 \mathrm{~Gy} / 25 \mathrm{fr})$ plus either CF with dose reduction or 5-fluorouracil monotherapy \\
Palliative (1st line) & CF & CF with dose reduction or 5-fluorouracil or S-1 monotherapy or BSC \\
Palliative (2nd line) & Nivolumab (biweekly) & Nivolumab (every 3 or more weeks) or BSC \\
Palliative (3rd line) & Paclitaxel or Docetaxel or BSC & Paclitaxel with less frequent dosing intervals or Docetaxel with G-CSF or BSC \\
\hline
\end{tabular}

Risk factors associated with COVID-19 are: age ( $\geq 75$ years), performance status $\geq 2$, comorbidity (hypertension, diabetes mellitus, liver or renal or lung dysfunction). These recommendations are based on single institutional experience

$C F$ cisplatin plus 5 -fluorouracil; $R T$ radiotherapy; $B S C$ best supportive care; $G$ - $C S F$ granulocyte-colony stimulating factor

${ }^{a}$ Low risk is defined as the presence of no risk factor

${ }^{\mathrm{b}} \mathrm{High}$ risk is defined as the presence of one or more risk factors 
the current standard adjuvant chemotherapy [38]. Although the interim analysis of the JACCRO-GC 07 trial showed that the superiority of DS compared with S-1 monotherapy in terms of 3-year RFS, the improvement in overall survival (OS) remains unclear. In addition, severe (grade 3/4) neutropenia was observed twice as often in the DS (38.1\%) than in the S-1 (16.1\%) patients [42].

Furthermore, the ACTS-GC trial did not prove that S-1 was effective as adjuvant chemotherapy for patients aged older than 80 years [3]. Taken together, regarding indications of adjuvant chemotherapy, S-1 alone for Stage III GC patients with high risk of COVID-19 infection is recommended and adjuvant chemotherapy for elderly patients (>80 years) is not recommended. Patients receiving ongoing adjuvant chemotherapy and without severe hematologic toxicity before the onset of the COVID-19 pandemic should continue receiving that same treatment during the pandemic; however, dose reduction or stopping should be considered for patients with high risk of COVID-19 infection.

\section{Palliative chemotherapy}

In principle, we recommend continuing of the administration of the same standard chemotherapy to patients with low risk of COVID-19 infection, even during the COVID-19 pandemic. However, reducing the frequency of hospital visits by modifying treatment schedules (such as reducing the intensity of chemotherapy, delaying treatment or skipping cycles) for patients with high risk of COVID-19 infection may be considered.

As first-line chemotherapy, for patients with high tumor burden or tumor-related signs/symptoms or low risk of
COVID-19 infection, combination of fluoropyrimidine and oxaliplatin (if HER2 is positive, plus trastuzumab) is recommended as a standard regimen [38]. For patients with cytology-positive (CY1) tumors who were considered for conversion surgery, the treatment intensity should be maintained. On the other hand, for patients with low tumor burden or high risk of COVID-19 infection, non-intensive monotherapy [S-1 plus oxaliplatin (SOX)] with reduced dose of oxaliplatin, S-1 monotherapy, or 5-FU/leucovorin) is an alternative regimen. Prescription for two courses at once will be allowed for patients who received S-1 monotherapy as maintenance therapy to reduce the frequency of their hospital visits.

As second line therapy, ramucirumab plus paclitaxel / nanoparticle albumin-bound (nab)-paclitaxel is the standard of care in Japan [38, 43, 44]. We propose skipping the administration of paclitaxel/nab-paclitaxel at day 8 to avoid neutropenia and to reduce the frequency of hospital visits. A previously published study has indicated that biweekly administration of ramucirumab plus paclitaxel did not compromise efficacy [45].

As third-line therapy, the subgroup analysis of Japanese patients in the ATTRACTION-2 trial reported a very low frequency of immune-related pneumonitis $(n=1,0.7 \%$ of 152 patients) [46]. Therefore, nivolumab is recommended in third or later line, but also consider prolonging the interval between administration (3-6 weeks) for responders. Irinotecan and trifluridine/tipiracil are also third-line or later treatment options for GC [47, 48]. However, these two agents show a relatively high risk of severe hematologic toxicity (>30\%) [47, 48]. Thus, the treatment modification (reducing the intensity of chemotherapy, delaying treatment, skipping

Table 3 Principles of chemotherapy for gastric cancer according to each risk group in the COVID-19 pandemic

\begin{tabular}{|c|c|c|}
\hline Treatment settings & Low risk ${ }^{\mathrm{a}}$ & High risk $^{\mathrm{b}}$ \\
\hline Neoadjuvant & SOX (for only bulky N) & Unrecommended \\
\hline Adjuvant & $\begin{array}{l}\text { Stage II: S-1 } \\
\text { Stage III: DS }\end{array}$ & StageII/III: $75-79$ y/o: S-1 with reduced dose, $\geq 80$ y/o: surgery alone \\
\hline Palliative (1st line) & $\mathrm{SOX} \pm \mathrm{Tmab}^{\mathrm{c}}$ & $\begin{array}{l}\mathrm{SOX} \pm \mathrm{Tmab}^{\mathrm{c}} \text { with delay interval of reduced dose of SOX or S-1 } \pm \mathrm{Tmab}^{\mathrm{c}} \\
\text { with reduced dose of S-1 }\end{array}$ \\
\hline Palliative (2nd line) & PTX/nabPTX \pm RAM (bi-weekly) & $\begin{array}{l}\text { PTX/nabPTX } \pm \text { RAM (with delay interval of bi-weekly) or PTX/nabPTX } \\
\text { (with delay interval of bi-weekly) or BSC }\end{array}$ \\
\hline Palliative (3rd line or later) & Nivolumab or FTD/TPI or IRI & $\begin{array}{l}\text { Nivolumab (with delay interval) or FTD/TPI with delay interval or reduced } \\
\text { dose or IRI with delay interval of reduced dose or RAM alone or BSC }\end{array}$ \\
\hline
\end{tabular}

Risk factors associated with COVID-19 are: age ( $\geq 75$ years), performance status $\geq 2$, comorbidity (hypertension, diabetes mellitus, liver or renal or lung dysfunction). These recommendations are based on single institutional experience

SOX S-1 plus oxaliplatin; DS docetaxel plus S-1; Tmab trastuzumab; PTX paclitaxel; RAM ramucirumab; BSC best supportive care; FTD/TPI trifluridine/tipiracil; IRI Irinotecan

${ }^{a}$ Low risk is defined as the presence of no risk factor

${ }^{b}$ High risk is defined as the presence of one or more risk factors

${ }^{\mathrm{c}} \mathrm{HER} 2$ positive case: + Tmab 
cycles, or stopping) should be considered, especially for patients with high-risk factors of COVID-19 infection.

Because it is associated with less hematologic toxicity than irinotecan and trifluridine/tipiracil, ramucirumab monotherapy could also be a treatment option for patients with high risk of COVID-19 infection [49, 50].

Table 3 lists the principles of chemotherapy for GC according to each risk group in the COVID-19 pandemic.

\section{Colorectal cancer}

\section{Neoadjuvant chemotherapy (including chemoradiotherapy)}

For patients with low risk of COVID-19 infection, we should start and continue standard neoadjuvant chemotherapy (chemoradiotherapy), if possible. However, shortcourse radiotherapy $(5 \times 5 \mathrm{~Gy})$ followed by delayed surgery may be the best option, except for $\mathrm{T} 4$ rectal cancer. It has been demonstrated that the pathological complete response rate was significantly higher in patients whose surgery was delayed than in patients who underwent immediate surgery, although there were no differences in rates of preservation of sphincters and negative margins of resected tumors between two groups [51]. Neoadjuvant chemotherapy is not recommended for patients with high risk of COVID-19 infection.

\section{Adjuvant chemotherapy}

For patients with low risk of COVID-19 infection, we should start and continue standard adjuvant chemotherapy if possible (T3 and N1: capecitabine plus oxaliplatin (CapeOx) 3-6 months, T4 and/or N2: CapeOX 6 months) [52].
However, adjuvant chemotherapy that reduces the intensity and shortens the duration of chemotherapy is an alternative option. Treatment should be performed at a local hospital near where a patient resides if traveling to the cancer center is too difficult.

For patients with high risk of COVID-19 infection, adjuvant chemotherapy is not recommended. Fluoropyrimidine monotherapy is the alternative option of the adjuvant chemotherapy.

For patients with microsatellite instability high (MSI$\mathrm{H}) /$ deficient mismatch repair (dMMR), patients receiving adjuvant chemotherapy for stages II and III MSI-H/dMMR CRC have a lower recurrence rate than MSS/pMMR CRC patients, and also a favorable prognosis [53].

\section{Palliative chemotherapy}

We should start and continue standard chemotherapy according to the National Comprehensive Cancer Network $(\mathrm{NCCN}) / \mathrm{ESMO} / \mathrm{Japanese}$ Society for Cancer of the Colon and Rectum (JSCCR) guidelines for patients with metastatic $\mathrm{CRC}$ and high tumor burden or who expect to undergo conversion surgery, [54-56] if possible. Furthermore, nonintensive chemotherapies such as fluoropyrimidines (5-FU, capecitabine and S-1) and bevacizumab, fluoropyrimidine monotherapy, or a stop-and-go strategy are alternative options.

For patients with a low tumor burden or high-risk factors, non-intensive chemotherapy may be desirable. Furthermore, delaying treatment and treating at the patient's local hospital should be considered if traveling to the cancer center is too difficult.

Table 4 Principles of chemotherapy for colorectal cancer according to each risk group in the COVID-19 pandemic

\begin{tabular}{|c|c|c|c|}
\hline Treatment setting & High tumor burden/ Low risk ${ }^{\mathrm{a}}$ & Low tumor burden/high risk ${ }^{\text {b }}$ & MSI-H/dMMR \\
\hline Neoadjuvant & $\begin{array}{l}\text { Standard chemotherapy/chemoradiotherapy (alternative: } \\
\text { short course RT) }\end{array}$ & Unrecommended & $\begin{array}{l}\text { Depends on } \\
\text { tumor burden } \\
\text { and risk factors }\end{array}$ \\
\hline Adjuvant & $\begin{array}{l}\text { Standard chemotherapy, 3-6 months (alternative: replace- } \\
\text { ment of 5-FU to capecitabine, option: FU monotherapy, } \\
\text { treatment at the local hospitals) }\end{array}$ & Unrecommended (option: FU monotherapy) & Unrecommended \\
\hline Palliative & $\begin{array}{l}\text { Standard chemotherapy (alternative: replacement of 5-FU to } \\
\text { capecitabine, option: non-intensive chemotherapy) }\end{array}$ & $\begin{array}{l}\text { Non-intensive chemotherapy (option: } \\
\text { treatment delay, treatment at the local } \\
\text { hospitals) }\end{array}$ & $\begin{array}{l}\text { Standard } \\
\text { chemotherapy } \\
\text { or Pembroli- } \\
\text { zumab }\end{array}$ \\
\hline
\end{tabular}

Risk factors associated with COVID-19 are: age ( $\geq 75$ years), performance status $\geq 2$, comorbidity (hypertension, diabetes mellitus, liver or renal or lung dysfunction). These recommendations are based on single institutional experience

$M S I-H$ microsatellite instability high, $d M M R$ deficient mismatch repair, $R T$ radiotherapy, FU montherapy: capecitabine, S-1, tegafur-uracil/Leucovorin for 6 months, Non-intensive chemotherapy: Fluoropyrimidine and bevacizumab, Fluoropyrimidine monotherapy or a stop-and-go strategy

${ }^{a}$ Low risk is defined as the presence of no risk factor

${ }^{\mathrm{b}} \mathrm{High}$ risk is defined as the presence of one or more risk factors 
We consider using pembrolizumab for patients with MSI-H/dMMR regardless of risk factors associated with severe COVID-19 [57], although there are several controversial issues, such as the differential diagnosis between COVID-19- related lung injury and ICI-associated immune-related adverse events and the risk of worsening lung injury by activating the immune response.

Table 4 lists the principles of chemotherapy for CRC according to each risk group in the COVID-19 pandemic.

\section{Biliary-pancreatic cancer}

\section{Pancreatic cancer}

\section{Adjuvant chemotherapy}

The survival benefit of S-1 monotherapy as adjuvant chemotherapy for PC has been shown by the JASPAC-01 study [58]. The recent results of the Prep-02/JSAP05 trial have revealed the survival benefit of gemcitabine plus S-1 (GS) as neoadjuvant chemotherapy for resectable PC [59]. Based on these results, NAC with GS and postoperative adjuvant chemotherapy with S-1 have become the standard of care for patients with resectable PC in Japan.

Considering the poor outcome of treating PC with surgery alone and the significant survival-prolonging effect of adjuvant (neoadjuvant) chemotherapy, NAC with GS and postoperative adjuvant chemotherapy with S-1 are recommended even during the COVID-19 pandemic. The initiation of adjuvant chemotherapy can be delayed until 12 weeks after surgery if the patient does not recover sufficiently after surgery. FOLFIRINOX is not recommended as postoperative adjuvant chemotherapy, because severe neutropenia has been reported, and it is not covered by insurance in Japan.

\section{Palliative chemotherapy}

There is no indication for discontinuing or changing the current chemotherapy in patients with low risk of COVID19 infection. However, if a new treatment is planned, gemcitabine plus nab-paclitaxel, which has a relatively low incidence of hematologic toxicity, is recommended [60]. FOLFOX may be considered in cases in which platinum is preferred, such as in patients with breast cancer gene (BRCA) mutations [61].

For elderly patients or those with high risk of COVID-19 infection, changes in treatment intervals or a change to $\mathrm{S}-1$ monotherapy [62,63] may be considered.

For patients undergoing gemcitabine plus nab-paclitaxel, the deletion of day 8 of the standard regimen, namely, providing infusions of both drugs on days 1 and 15 of each 28-day cycle, should be considered to avoid neutropenia and to reduce the frequency of hospital visits. Some studies have reported that this approach improves patient tolerance without significantly compromising efficacy $[64,65]$.

Concerning second-line treatment, we need to evaluate the indications for the treatment carefully for not only new patients but also patients currently undergoing treatment. Best supportive care can be an option as the situation demands.

\section{Biliary tract cancer}

\section{Adjuvant chemotherapy}

In Japan, there is no evidence of improved survival for biliary tract cancer patients treated with adjuvant or neoadjuvant chemotherapy. Adjuvant/neoadjuvant chemotherapy should not be administered to biliary tract cancer patients during the COVID-19 pandemic.

\section{Palliative chemotherapy}

There is no indication for discontinuing or changing current chemotherapy in patients with low risk of COVID-19 infections. Gemcitabine plus cisplatin is the standard of care for patients with unresectable biliary tract cancer [66]. Intervals between chemotherapy cycles, such as a biweekly regimen, should be lengthened as the situation demands because gemcitabine plus cisplatin sometimes causes severe neutropenia.

Table 5 Principles of chemotherapy for biliary-pancreatic cancer according to each risk group in the COVID-19 pandemic

\begin{tabular}{lll}
\hline Treatment settings & Low risk $^{\mathrm{a}}$ & High risk $^{\mathrm{b}}$ \\
\hline $\begin{array}{lll}\text { Pancreatic cancer } \\
\text { Neoadjuvant }\end{array}$ & GS & GS \\
$\begin{array}{l}\text { Adjuvant } \\
\text { Palliative (1st line) }\end{array}$ & GnP & S-1 \\
& & GnP or S-1 monotherapy \\
or BSC \\
Palliative (2nd line) & S-1 & BSC \\
Biliary tract cancer & & \\
Neoadjuvant & Unrecommended & Unrecommended \\
Adjuvant & Unrecommended & Unrecommended \\
Palliative (1st line) & GC & Gem monotherapy or S-1 \\
& & or BSC \\
Palliative (2nd line) & S-1 & Unrecommended \\
\hline
\end{tabular}

Risk factors associated with COVID-19 are: age ( $\geq 75$ years), performance status $\geq 2$, comorbidity (hypertension, diabetes mellitus, liver or renal or lung dysfunction). These recommendations are based on single institutional experience

$G S$ gemcitabine plus S-1; GnP gemcitabine plus nab-paclitaxel; $B S C$ best supportive care; $G C$ gemcitabine plus Cisplatin

${ }^{a}$ Low risk is defined as the presence of no risk factor

${ }^{\mathrm{b}} \mathrm{High}$ risk is defined as the presence of one or more risk factors 
Table 5 lists the principles of chemotherapy used for biliary-pancreatic cancer according to each risk group in the COVID-19 pandemic.

\section{Discussion}

In this article, we outlined the recommendations for making decisions on chemotherapies for GI cancers during the COVID pandemic. There are several recommendations and guidelines already published worldwide (16-21). However, few specific recommendations on chemotherapy have been issued for patients with GI cancers. Moreover, practices differ in many aspects in GI cancers between US/Europe and Japan (e.g., oral agent such as S-1 are widely used). And the guidelines from Japan are general and do not specifically address how to modify the treatment but maintain effectiveness as this article described. In this respect, we believe that our guidance is based on actual clinical practice of GI oncology in Japan and would be useful not only to cancer center institutions but also to general hospitals.

Now, providing normal medical care is also being somewhat limited because of COVID-19. The standard approach for GI cancer, early detection and early treatment, such as that of endoscopic screening and endoscopic submucosal dissection (ESD), respectively, which have been very prevalent in Japanese society, is in a crisis. In addition, compared to other countries, a higher number of Japanese patients have received subsequent chemotherapy after disease progression [67]. This strategy of early detection and treatment and higher rates of subsequent implementation of chemotherapy might have contributed to longer survival in Japanese patients than the survival of other populations. However, retaining a similar treatment strategy after the onset of the COVID-19 pandemic might be difficult.

Despite these difficulties, we must continue to administer chemotherapy to the patients who need it. The most important goal of treatment was to complete the protocol without reducing the intensity of treatment, especially if cure is desired. In the palliative setting, however (in fact, the majority of cases are in the palliative setting), minimizing the risk of developing severe COVID-19 that is associated with chemotherapy and maximizing survival and symptom relief are important. In order to achieve these important goals, the duration of treatments should be modified; and dose reduction, treatments with reduced toxicity, change to oral agents such as S-1 that require reduced visits to the cancer center, and empirical use of granulocyte-stimulating factor should be considered.

Along with protecting the patient from infection, infection of healthcare providers must be avoided. If healthcare providers are infected, and the medical supply system is not maintained, anticancer treatments must be reduced. For example, our department has created a business continuity plan that limits the number of treatments based on proportion of staff that is absent because of COVID-19 infection. Such preparations are important for any medical institution.

With regard to ongoing clinical trials, with the considerations for protecting patients and the institutional standards for Good Clinical Practice (GCP) during the COVID-19 pandemic state, recruitment of new patients was avoided as own consensus in our institution, and the safety for the current participants should be prioritized. Guidance is also provided by the Food and Drug Administration [68] and referring to it is recommended.

The limitation of this guidance is that our recommendations are not comprehensively evidence-based and are largely based on expert opinion. We have just started using it in our institution and its feasibility should be confirmed. Currently, several issues on COVID-19 and cancer chemotherapy have been published in addition to ours, but all of them remain controversial. The accumulation of evidencebased data should lead to the establishment of optimal treatment policies. As effective treatments and vaccines for COVID-19 are developed, this guidance may need updating, and there will be additional new issues to consider, such as the risks associated with concomitant use of vaccine against COVID-19 and ICI therapy. Staying updated with new information for the treatments of GI cancers is important.

In summary, we in the field of GI oncology recommend considering modifications to conventional chemotherapy that are based on the prevalence of COVID-19, treatment goals and patients' vulnerabilities. Even if the pandemic is successfully suppressed, subsequent pandemics will inevitably occur. These recommendations should, therefore, be useful in the post-Corona era as well.

\section{Compliance with ethical standards}

Conflict of interest Daisuke Takahari has received lecture fees from Taiho Pharmaceutical Co.,Ono Pharmaceutical Co., Eli Lilly Japan, and Bristol-Myers Squibb. Eiji Shinozaki has received lecture fee from Eli Lilly Japan. Masato Ozaka has received lecture fees from Yakult Honsha Co., Ltd. and Taiho Pharmaceutical Co. Kensei Yamaguchi has received research grants and lecture fees from Taiho Pharmaceutical Co., Eli Lilly Japan, Ono Pharmaceutical Co., Bristol-Myers Squibb, Yakult Honsha Co., Ltd., Sanofi and Chugai Pharmaceutical Co., Ltd., and lecture fees from Takeda Pharmaceutical Co. Ltd, Bayer, Daiichi-Sankyo, Dainippon-Sumitomo Pharma, Merck Sharp and Dohme, and Gilead Sciences, Inc. Takeru Wakatsuki, Akira Ooki, Takeshi Suzuki, Izuma Nakayama, Hiroki Osumi, Daisaku Kamiimabeppu, Taro Sato, Mariko Ogura, Mitsukuni Suenaga and Keisho Chin have no conflict of interest.

Open Access This article is licensed under a Creative Commons Attribution 4.0 International License, which permits use, sharing, adaptation, distribution and reproduction in any medium or format, as long as you give appropriate credit to the original author(s) and the source, 
provide a link to the Creative Commons licence, and indicate if changes were made. The images or other third party material in this article are included in the article's Creative Commons licence, unless indicated otherwise in a credit line to the material. If material is not included in the article's Creative Commons licence and your intended use is not permitted by statutory regulation or exceeds the permitted use, you will need to obtain permission directly from the copyright holder. To view a copy of this licence, visit http://creativecommons.org/licenses/by/4.0/.

\section{References}

1. World Health Organization (2020) Coronavirus Disease (COVID19) Outbreak; 2020. https://www.who.int/emergencies/diseases/ novel- coronavirus-2019. Accessed 18 Mar 2020.

2. Johns Hopkins University (2020) COVID-19 Dashboard by the Center for Systems Science and Engineering at Johns Hopkins University. https://gisanddata.maps.arcgis.com/apps/opsdashboa rd/index. Accessed 20 May 2020.

3. Ministry of Health, Labor and Health Care Japan (2020) https:// www.mhlw.go.jp/stf/seisakunitsuite/bunya/0000164708_00001 .html\#kokunaihassei. Accessed 20 May 2020.

4. Ministry of Health, Labor and Health Care Japan (2020) https:// www.mhlw.go.jp/content/10900000/000620733pdf. Accessed 18 Mar 2020.

5. Ministry of Health, Labor and Health Care Japan (2020) https:// www.mhlw.go.jp/content/10900000/000624195pdf. Accessed 18 Mar 2020.

6. Liang W, Guan W, Chen R et al (2020) Cancer patients in SARSCoV-2 infection: a nationwide analysis in China. Lancet Oncol 21:335-337

7. Xia Y, Jin R, Zhao J et al (2020) Risk of COVID-19 for patients with cancer. Lancet Oncol 21:e180

8. Guan WJ, Liang WH, Zhao Y et al (2020) Comorbidity and its impact on 1590 patients with Covid-19 in China: a nationwide analysis. Eur Respir J 55:2000547

9. Wang B, Li R, Lu Z et al (2020) Does comorbidity increase the risk of patients with COVID-19: evidence from meta-analysis. Aging (Albany NY) 12:6049-6057

10. World Health Organization (2020) Report of the WHO-China Joint Mission on Coronavirus Disease 2019 (COVID-19). WHO, Geneva

11. Dai M, Liu D, Liu M et al (2020) Patients with cancer appear more vulnerable to SARS-COV-2: a multicenter study during the COVID-19 outbreak. Cancer Discov 10:783-791

12. Deng G, Yin M, Chen X et al (2020) Clinical determinants for fatality of 44,672 patients with COVID-19. Crit Care 24:179

13. Miyashita H, Mikami T, Chopra N et al (2020) Do patients with cancer have a poorer prognosis of COVID-19? an experience in New York city. Ann Oncol 31:1088

14. Onder G, Rezza G, Brusaferro S (2020) Case-fatality rate and characteristics of patients dying in relation to COVID-19 in Italy. JAMA March 23. Online ahead of print

15. Mehta V, Goel S, Kabarriti R et al (2020) Case fatality rate of cancer patients with COVID-19 in a New York hospital system. Cancer Discov. https://doi.org/10.1001/jama.2020.4683

16. American Society of Clinical Oncology (2020) COVID-19 Patient Care Information. https://www.asco.org/asco-coronavirus-infor mation/care-individuals-cancer-during-covid-19. Accessed 7 May 2020.

17. European Society of Medical Oncology (2020) COVID-19: supporting oncology professionals. https://www.esmo.org/covid -19-and-cancer/supporting-oncology-professionals. Accessed 7 May 2020.
18. Schrag D, Hershman DL, Basch E (2020) Oncology practice during the COVID-19 pandemic. JAMA. https://doi.org/10.1001/ jama.2020.6236

19. You B, Ravaud A, Canivet A et al (2020) The official French guidelines to protect patients with cancer against SARS-CoV-2 infection. Lancet Oncol 21:619-621

20. Pinto C, Cagnazzo C (2020) Indications regarding the management of interventional clinical trials with drugs during the current COVID-19 emergency in Italy. ESMO Open 5:e000782

21. Japanese Cancer Society (2020) The Japanese Society of Clinical Oncology, and the Japanese Society of Medical Oncology. https ://www.jca.gr.jp/public/c_q_and_a.html Accessed 15 May 2020.

22. Lou E, Beg S, Bergsland E et al (2020) Modifying practices in GI oncology in the face of COVID-19: recommendations from expert oncologists on minimizing patient risk. JCO Oncol Pract. https:// doi.org/10.1200/OP.20.00239

23. Morens DM, Taubenberger JK, Fauci AS et al (2008) Predominant role of bacterial pneumonia as a cause of death in pandemic influenza: implications for pandemic influenza preparedness. J Infect Dis 198:962-970

24. Lodigiani C, Iapichino G, Carenzo L et al (2020) Venous and arterial thromboembolic complications in COVID-19 patients admitted to an academic hospital in Milan, Italy. Thromb Res 191:9-14

25. Mehra MR, Desai SS, Kuy S et al (2020) Cardiovascular disease, drug therapy, and mortality in Covid-19. N Engl J Med 382(25):e102. https://doi.org/10.1056/NEJMoa2007621 [Retraction in: N Engl J Med 2020]

26. Vaduganathan M, Vardeny O, Michel T et al (2020) Renin-angiotensin-aldosterone system inhibitors in patients with Covid-19. N Engl J Med 382:1653-1659

27. Li J, Wang X, Chen J et al (2020) Association of renin-angiotensin system inhibitors with severity or risk of death in patients with hypertension hospitalized for Coronavirus Disease 2019 (COVID-19) infection in Wuhan, China. JAMA Cardiol. https:// doi.org/10.1001/jamacardio.2020.1624

28. Ando N, Kato H, Igaki H et al (2012) A Randomized trial comparing postoperative adjuvant chemotherapy with cisplatin and 5-fluorouracil versus preoperative chemotherapy for localized advanced squamous cell carcinoma of the thoracic esophagus (JCOG9907). Ann Surg Oncol 19:68-74

29. Kitagawa Y, Uno T, Oyama T et al (2019) Esophageal cancer practice guidelines 2017 edited by the Japan Esophageal Society: part 1. Esophagus 16:1-24

30. Yokota T, Hatooka S, Ura T et al (2011) Docetaxel plus 5-fluorouracil and cisplatin (DCF) induction chemotherapy for locally advanced borderline-resectable T4 esophageal cancer. Anticancer Res 31:3535-3541

31. Yokota T, Kato K, Hamamoto Y et al (2016) Phase II study of chemoselection with docetaxel plus cisplatin and 5-fluorouracil induction chemotherapy and subsequent conversion surgery for locally advanced unresectable oesophageal cancer. Br J Cancer 115:1328-1334

32. Ando N, Iizuka T, Ide H et al (2003) Surgery plus chemotherapy compared with surgery alone for localized squamous cell carcinoma of the thoracic esophagus: a Japan Clinical Oncology Group Study-JCOG9204. J Clin Oncol 21:4592-4596

33. Kato K, Cho BC, Takahashi $M$ et al (2019) Nivolumab versus chemotherapy in patients with advanced oesophageal squamous cell carcinoma refractory or intolerant to previous chemotherapy (ATTRACTION-3): a multicentre, randomised, open-label, phase 3 trial. Lancet Oncol 20:1506-1517

34. Kato H, Sato A, Fukuda H et al (2009) A phase II trial of chemoradiotherapy for stage i esophageal squamous cell carcinoma: Japan Clinical Oncology Group Study (JCOG9708). Jpn J Clin Oncol 39:638-643 
35. Shinoda M, Ando N, Kato K et al (2015) Randomized study of low-dose versus standard-dose chemoradiotherapy for unresectable esophageal squamous cell carcinoma (JCOG0303). Cancer Sci 106:407-412

36. Minsky BD, Pajak TF, Ginsberg RJ et al (2002) INT 0123 (Radiation Therapy Oncology Group 94-05) phase III trial of combined-modality therapy for esophageal cancer: highdose versus standard-dose radiation therapy. J Clin Oncol 20:1167-1174

37. Zaorsky NG, Yu JB, McBride SM et al (2020) Prostate cancer radiotherapy recommendations in response to COVID-19. Adv Radiat Oncol. https://doi.org/10.1016/j.adro.2020.03.010

38. Japanese Gastric Cancer Association (2020) Japanese gastric cancer treatment guidelines 2018 (5th edition). Gastric Cancer. https ://doi.org/10.1007/s10120-020-01042-y

39. Tsuburaya A, Mizusawa J, Tanaka Y et al (2014) Neoadjuvant chemotherapy with S-1 and cisplatin followed by D2 gastrectomy with para-aortic lymph node dissection for gastric cancer with extensive lymph node metastasis. Br J Surg 101:653-660

40. Sakuramoto S, Sasako M, Yamaguchi T et al (2007) Adjuvant chemotherapy for gastric cancer with S-1, an oral fluoropyrimidine. N Engl J Med 357:1810-1820

41. Sasako M, Sakuramoto S, Katai H et al (2011) Five-year outcomes of a randomized phase III trial comparing adjuvant chemotherapy with S-1 versus surgery alone in stage II or III gastric cancer. J Clin Oncol 29:4387-4393

42. Yoshida K, Kodera Y, Kochi M et al (2019) Addition of docetaxel to oral fluoropyrimidine improves efficacy in patient with Stage III gastric cancer: Interim analysis of JACCRO GC-07, a randomized controlled trial. J Clin Oncol 37:1296-1304

43. Wilke H, Muro K, Van Cutsem E et al (2014) Ramucirumab plus paclitaxel versus placebo plus paclitaxel in patients with previously treated advanced gastric or gastro-oesophageal junction adenocarcinoma (RAINBOW): a double-blind, randomised phase 3 trial. Lancet Oncol 15:1224-1235

44. Bando H, Shimodaira H, Fujitani K et al (2018) A phase II study of nab-paclitaxel in combination with ramucirumab in patients with previously treated advanced gastric cancer. Eur J Cancer 91:86-91

45. Rogers JE, Xiao L, Amlashi FG et al (2019) Ramucirumab and paclitaxel administered every 2 weeks (mRAINBOW Regimen) in advanced gastroesophageal adenocarcinoma. Oncology 96:252-258

46. Kato K, Satoh T, Muro K et al (2019) A subanalysis of Japanese patients in a randomized, double-blind, placebo-controlled, phase 3 trial of nivolumab for patients with advanced gastric or gastroesophageal junction cancer refractory to, or intolerant of, at least two previous chemotherapy regimens (ONO-4538-12, ATTRAC TION-2). Gastric Cancer 2019:344-354

47. Shitara K, Doi T, Dvorkin M et al (2018) Trifluridine/tipiracil versus placebo in patients with heavily pretreated metastatic gastric cancer (TAGS): a randomised, double-blind, placebo-controlled, phase 3 trial. Lancet Oncol 19:1437-1448

48. Makiyama A, Arimizu K, Hirano G et al (2018) Irinotecan monotherapy as third-line or later treatment in advanced gastric cancer. Gastric Cancer 21(3):464-472

49. Fuchs CS, Tomasek J, Yong CJ et al (2014) Ramucirumab monotherapy for previously treated advanced gastric or gastro-oesophageal junction adenocarcinoma (REGARD): an international, randomised, multicentre, placebo-controlled, phase 3 trial. Lancet 383:31-39

50. Murahashi S, Takahari D, Wakatsuki T et al (2018) A retrospective analysis of ramucirumab monotherapy in previously treated Japanese patients with advanced or metastatic gastric adenocarcinoma. Int J Clin Oncol 23:92-97

51. Bujko K, Partycki M, Pietrzak L (2014) Neoadjuvant radiotherapy $(5 \times 5$ Gy): immediate versus delayed surgery. Recent Results Cancer Res 203:171-187
52. Grothey A, Sobrero AF, Shields AF et al (2018) Duration of adjuvant chemotherapy for stage III colon cancer. N Engl J Med 378:1177-1188

53. Des Guetz G, Schischmanoff O, Nicolas P et al (2009) Does microsatellite instability predict the efficacy of adjuvant chemotherapy in colorectal cancer? A systematic review with metaanalysis. Eur J Cancer 45:1890-1896

54. Benson AB, Venook AP, Al-Hawary MM et al (2018) NCCN guidelines insights: colon cancer, version 2.2018. J Natl Compr Canc Netw 16:359-369

55. Yoshino T, Arnold D, Taniguchi $\mathrm{H}$ et al (2018) Pan-Asian adapted ESMO consensus guidelines for the management of patients with metastatic colorectal cancer: a JSMO-ESMO initiative endorsed by CSCO, KACO, MOS, SSO and TOS. Ann Oncol 29:44-70

56. Hashiguchi Y, Muro K, Saito Y et al (2020) Japanese Society for Cancer of the Colon and Rectum (JSCCR) guidelines 2019 for the treatment of colorectal cancer. Int J Clin Oncol 25:1-42

57. Overman MJ, McDermott R, Leach JL et al (2017) Nivolumab in patients with metastatic DNA mismatch repair-deficient or microsatellite instability-high colorectal cancer (CheckMate 142): an open-label, multicentre, phase 2 study. Lancet Oncol 18:1182-1191

58. Uesaka K, Boku N, Fukutomi A et al (2016) Adjuvant chemotherapy of S-1 versus gemcitabine for resected pancreatic cancer: a phase 3, open-label, randomised, non-inferiority trial (JASPAC 01). Lancet 388:248-257

59. Motoi F, Kosuge T, Ueno H et al (2019) Randomized phase II/ III trial of neoadjuvantchemotherapy with gemcitabine and S-1 versus upfront surgery for resectable pancreatic cancer( Prep-02/ JSAP- 05). Jpn J Clin Oncol 49:190-194

60. Von Hoff DD, Ervin T, Arena FP et al (2013) Increased survival in pancreatic cancer with nab-paclitaxel plus gemcitabine. N Engl J Med 369:1691-1703

61. Fogelman D, Sugar EA, Oliver G et al (2015) Family history as a marker of platinum sensitivity in pancreatic adenocarcinoma. Cancer Chemother Pharmacol 76:489-498

62. Todaka A, Fukutomi A, Boku N et al (2010) S-1 monotherapy as second-line treatment for advanced pancreatic cancer after gemcitabine failure. Jpn J Clin Oncol 40:567-572

63. Sudo K, Yamaguchi T, Nakamura K et al (2011) Phase II study of S-1 in patients with gemcitabine-resistant advanced pancreatic cancer. Cancer Chemother Pharmacol 67:249-254

64. Ahn DH, Krishna K, Blazer M et al (2017) A modified regimen of biweekly gemcitabine and nab-paclitaxel in patients with metastatic pancreatic cancer is both tolerable and effective: a retrospective analysis. Ther Adv Med Oncol 9:75-78

65. Kokkali S, Tripodaki E-S, Drizou M et al (2018) Biweekly gemcitabine/nab-paclitaxel as first-line treatment for advanced pancreatic cancer. Vivo 32:653-657

66. Valle J, Wasan H, Palmer DH et al (2010) Cisplatin plus gemcitabine versus gemcitabine for biliary tract cancer. N Engl J Med 362:1273-1281

67. Sawaki A, Yamada Y, Yamaguchi K et al (2018) Regional differences in advanced gastric cancer: exploratory analyses of the AVAGAST placebo arm. Gastric Cancer 21:429-438

68. The Food and Drug Administration (2020) FDA Guidance on Conduct of Clinical Trials of Medical Products during COVID-19 Public Health Emergency. https://www.fda.gov/regulatory-infor mation/search-fda-guidance-documents/fda-guidance-conductclinical-trials-medical-products-during-covid-19-public-healt h-emergency. Accessed 20 May 2020.

Publisher's Note Springer Nature remains neutral with regard to jurisdictional claims in published maps and institutional affiliations. 